\title{
Editorial: Measuring Solar Magnetic Fields-An Outline of History, Current Status and Challenges
}

\author{
André Balogh ${ }^{1} \cdot$ Rudolf von Steiger ${ }^{2}$
}

Published online: 4 September 2017

(C) Springer Science+Business Media B.V. 2017

The variability in solar activity and its causes have remained the subject of theoretical and experimental investigations for several decades. The objective is to understand the causal chains that shape the near and far solar environment, from the interior of the Sun all the way to interplanetary space. The observational means as well as the theoretical and modelling resources devoted to the topic have been formidable. Built on a broad range of terrestrial and space-based observational resources, solar science has progressed to the point where there are vast quantities of observational data and very detailed models of phenomena that describe the processes in the interior of the Sun, its surface and atmosphere, as well as in the heliosphere that extends to the interstellar medium at a distance of more than $100 \mathrm{AU}$.

In parallel with the ever-increasing volume of observations and constantly refined and renewed models, there is a flow of publications, scientific papers and books, and there are numerous conferences and workshops yearly that are devoted to aspects of solar physics. Yet, despite the proliferation of phenomenological descriptions, there are many open questions concerning the physical processes underlying the causal chains of solar variability. Some of these have been enumerated and discussed, among others, by Parker in this journal (Vol. 144, 2009).

There is, however, general agreement on the key role played by magnetic fields in all solar phenomena and throughout the sun and its close and distant environment. Reviews of magnetic fields in the sun's interior, its atmosphere and in the heliosphere in that volume and elsewhere provide an overview of the place of magnetic fields in solar physics. The key variability manifests itself in the many phenomena related to the 11-year activity cycle, which was also reviewed in this journal (Vol. 186, 2014).

While the central role of magnetic fields is acknowledged, understanding the many processes that involve magnetic fields is hampered by limitations of their quantitative knowledge. Measuring magnetic fields remotely in the solar environment is difficult, even though

R. von Steiger

vsteiger@issibern.ch

Imperial College, London, UK

2 International Space Science Institute, Bern, Switzerland 
solar observers have always taken advantage of newly discovered techniques in their instruments. The first discovery of strong magnetic fields in sunspots by Hale in the early 20th century came from applying the then recently described Zeeman effect of magnetic fields on the splitting of spectral lines. Progress in solar physics has come about from increasing observational capabilities, outlined in the historical sequence of some of the reviews setting out progress in the field by Babcock and others. A detailed personal account of observing solar magnetic fields, with a fifty-year perspective, is given by Stenflo (this volume).

Helioseismology, the study of solar oscillations is a powerful tool for the study of the solar interior. Since the start of its use three decades ago, great progress has been made in measuring by inference solar magnetic fields in the interior of the sun, in the near-surface layers and in the photosphere. In particular, it has provided substantial observational support for refining global models of the solar dynamo-even though the debate concerning the "best" model remains open. This is one clear example of observational data prompting major advances in understanding the mechanisms at the origin of the sun's magnetic field and setting constraints on theoretical models, yet not being sufficiently comprehensive to enable a firm choice to be made among competing dynamo models.

Whole sun magnetographs were the first instruments, following the original observing instruments used by Hale, that allowed progressing beyond measurements of the strong magnetic fields associated with sunspots. The pioneering magnetograph constructed at the Mount Wilson Observatory by Babcock, carefully designed incorporating the best available technology, allowed an estimate of about $1 \mathrm{G}$ as the general surface magnetic field of the sun. He was also able to observe the polarity reversal of the sun associated with the sunspot cycle and propose, on the basis of his observations, a phenomenological model of the 22-year magnetic cycle that forms the first element of what is now known as the Babcock-Leighton model; for an up-to-date assessment of the status of that model in the current context, see Charbonneau in Living Reviews in Solar Physics.

Since the early days of magnetograph measurements in the 1950s, new generations of magnetographs and spectropolarimeters have considerably increased the resolution and general capabilities of solar surface magnetic measurements from ground-based observatories. It is now well established that the surface field is very highly non-uniform, with magnetic flux concentrated in narrow structures that represent the complex emergence of magnetic flux from below the photosphere.

The ability to fly a high-performance solar magnetograph on a space platform, such as the Solar Orbiter to be launched in 2018 will further increase the observational data base for improving the resolution and comprehensive coverage of solar surface magnetic measurements (see Kleint and Gandorfer, this volume). The resolution capability of the most recent observations of solar surface fields, now makes it possible to combine direct observations of photospheric fields to match the observed chromospheric fields. Such observations, made in this case with the Ultraviolet Filter Imager (SuFI) onboard the Sunrise balloonborne observatory, challenge the modelling capabilities by providing very tight constraints on the determination of very small scale magnetic field structures and their dynamics in the solar atmosphere. A broader based discussion of these observational capabilities and their interpretation is given by Borrero et al. (this volume).

In the solar photosphere, chromosphere and corona, the magnetic field measurements are generally very difficult. There are several measurement possibilities: Zeeman and Hanle spectropolarimetry of atomic line profiles, the structure and dynamics of visible plasma entrained in the magnetic field, and radio techniques. The principal challenge of spectropolarimetry is to devise an optimum strategy of sampling the spectropolarimetric hypercube, which spans the five dimensions $x, y, \lambda, S$, and $t$ (space, wavelength, Stokes vector, and 
time). Different strategies of sampling that hypercube and their relative merits and disadvantages are discussed by Lagg et al. (this volume). Our ability to make solar magnetic measurements critically depends on technology availability and understanding of radiative transfer in the solar atmosphere.

The in situ measurement of the magnetic field, where it can be performed in space, while accurate, suffers from the limitation of spatial coverage. Heliospheric magnetic field measurements have confirmed the solar origin of magnetic fields (in the heliosphere) and have narrowed the links between the Sun and the heliospheric medium. The first key results came quickly, in the 1960s - the relatively crude magnetometers (and spacecraft) performed well (if not always reliably). In particular, confirmation of the basic truth of Parker's model of coronal expansion and the consequent large-scale geometry of the magnetic field in the solar wind was achieved by the first few missions-Mariner 2, IMP 1 (and followers), and Mariners $4 \& 5$. The basic target of measuring fields of $\sim 5 \mathrm{nT}$ with accuracies better than $1 \mathrm{nT}$ was quickly achieved. New results since the early days have been achieved by a combination of the following factors: new trajectories through the heliosphere (in latitude and radial distance); improvements in magnetometers, but mostly in their data acquisition capabilities; an increased attention to achieve magnetically clean spacecraft in the 1970s, 1980s and 1990s (although nowadays agencies try workaround solutions that remain to be trusted and proved). The upcoming solar missions of ESA and NASA, Solar Orbiter and Solar Probe Plus (now aptly renamed Parker Solar Probe), respectively, both to be launched in 2018, will once again extend our knowledge vastly in the inner heliosphere down to ten solar radii.

As models of solar phenomena increase in complexity, thanks to improved understanding, a vastly increased computing capability and more refined observations covering broader parameter ranges, it is now more difficult to transfer measurement uncertainties and errors from the observables to the finer details of phenomenological models. Given also the increasing range of measurements and the need to combine observations and measurements from different sources, a good understanding of instrumental capabilities and limitations as well as quantitative error assessments have become necessary for confident modelling of complex solar magnetic phenomena.

Recognising this challenge, a Scientific Forum held at the International Space Science Institute in Bern, Switzerland on 20-21 November 2012 identified the potential benefits of assessing solar magnetic field measurements and how they can lead to a better understanding of solar phenomena. A Workshop was held in ISSI in February 2015 with the title Solar Magnetic Fields: From Measurements Towards Understanding. The collection of review papers, published in this volume of Space Science Reviews and the subsequent publication of the collection in the Space Science Series of ISSI (Vol. 57) provides a highly topical overview of the achievements of solar magnetic field measurements in improving the quantitative observational data base for making further progress in solar physics. In the same spirit, the Forum also recommended a broad review of the observables and parameters used to describe and explain the 11-year solar activity cycle. The collection of reviews arising from the Workshop held in ISSI in November 2013 was published in Space Science Reviews (Volume 186, 2014). 